\title{
Scattering of field-aligned beam ions upstream of Earth's bow shock
}

\author{
A. Kis ${ }^{1, *}$, M. Scholer ${ }^{1}$, B. Klecker ${ }^{1}$, H. Kucharek ${ }^{2}$, E. A. Lucek ${ }^{3}$, and H. Rème ${ }^{4}$ \\ ${ }^{1}$ Max-Planck-Institut für extraterrestrische Physik, Garching, Germany \\ ${ }^{2}$ Dept. of Physics and Institute for the Study of Earth, Oceans and Space, Univ. of New Hampshire, Durham, USA \\ ${ }^{3}$ Imperial College, London, UK \\ ${ }^{4}$ CESR, Toulouse, France \\ *now at: Geodetic and Geophysical Research Institute of HAS, Sopron, Hungary
}

Received: 10 July 2006 - Revised: 3 February 2007 - Accepted: 6 March 2007 - Published: 29 March 2007

\begin{abstract}
Field-aligned beams are known to originate from the quasi-perpendicular side of the Earth's bow shock, while the diffuse ion population consists of accelerated ions at the quasi-parallel side of the bow shock. The two distinct ion populations show typical characteristics in their velocity space distributions. By using particle and magnetic field measurements from one Cluster spacecraft we present a case study when the two ion populations are observed simultaneously in the foreshock region during a high Mach number, high solar wind velocity event. We present the spatialtemporal evolution of the field-aligned beam ion distribution in front of the Earth's bow shock, focusing on the processes in the deep foreshock region, i.e. on the quasi-parallel side. Our analysis demonstrates that the scattering of field-aligned beam (FAB) ions combined with convection by the solar wind results in the presence of lower-energy, toroidal gyrating ions at positions deeper in the foreshock region which are magnetically connected to the quasi-parallel bow shock. The gyrating ions are superposed onto a higher energy diffuse ion population. It is suggested that the toroidal gyrating ion population observed deep in the foreshock region has its origins in the FAB and that its characteristics are correlated with its distance from the FAB, but is independent on distance to the bow shock along the magnetic field.
\end{abstract}

Keywords. Interplanetary physics (Energetic particles; Planetary bow shocks) - Space plasma physics (Charged particle motion and acceleration; Wave-particle interactions)

\section{Introduction}

A well-known and long investigated feature of the Earth's bow shock is the presence and behavior of energetic ions in the foreshock region. The foreshock is the upstream re-

Correspondence to: M. Scholer

(mbs@mpe.mpg.de) gion which is magnetically connected to the bow shock and is dominated by waves and energized particles. The socalled upstream ions have energies from just above the solar wind bulk energy up to a few $100 \mathrm{keV}$. Based on their velocity space distribution characteristics the upstream ions have been divided into five groups. The diffuse ions, field-aligned beams and intermediate ions are basically gyrotropic and can be separated according to their pitch angle distributions. The other two distributions, the gyrating ions and the gyrophase bunched ions are nongyrotropic and they are discriminated from each other by their distance from the shock. As shown in previous studies by Scholer et al. (1981) and Ipavich et al. (1981), diffuse ions present a wide, mostly isotropic shelllike distribution in 3-D velocity space, with a flat energy spectrum extending well beyond $100 \mathrm{keV}$. These ions are accelerated at the quasi-parallel region of the bow shock, where $\Theta_{B n}$ (i.e., the angle between the interplanetary magnetic field and the bow shock normal) is less than $45^{\circ}$. It is known that diffuse ions are accompanied by MHD waves with frequencies less than the proton gyrofrequency (see e.g., Gosling et al., 1978; Bonifazi and Moreno, 1981a,b; Greenstadt et al., 1995; Russell and Farris, 1995). The field-aligned beam (FAB), composed of collimated ion beams with an energy of a few $\mathrm{keV}$, propagates along the interplanetary magnetic field away from the bow shock (Paschmann et al., 1980), (Schwartz et al., 1983), (Thomsen, 1985) and originates at regions of the bow shock where $\Theta_{B n}$ is between $45^{\circ}$ and $70^{\circ}$, i.e. at the quasi-perpendicular side. The statistical properties of the beam ions, like average number and energy density, were determined by Bonifazi and Moreno (1981a,b) based on a statistical analysis of ISEE 2 events.

In the transition region between the quasi-parallel and the quasi-perpendicular side the ion population was named "intermediate" by Paschmann et al. (1979), because this population has characteristics in velocity space intermediate between the diffuse and beam ion population. Gosling et al. (1982) and Paschmann et al. (1982) demonstrated that

Published by Copernicus GmbH on behalf of the European Geosciences Union. 
another group of ions, the so-called gyrating ions are shock reflected ions and are found in the close vicinity of the quasiperpendicular shock, i.e. within a distance of about one gyroradius. Because of their gyration around the magnetic field and due to the interplanetary magnetic field direction these ions quickly return to the shock front and are not able to move upstream into the foreshock region. The existence of a fifth ion group, the gyrophase bunched ions was first suggested by Hoshino and Terasawa (1985) on the basis of computer simulations. They were actually found at some distance from the shock (Thomsen, 1985; Fuselier et al., 1986) and are thought to be the result of FAB ions trapped in a self-generated monochromatic wave. In addition, Meziane et al. (2004) have reported observations of gyrophase bunched ions in the close vicinity of the quasi-parallel bow shock. These distributions are probably due to modulation of specularly reflected ions by the monochromatic low frequency upstream waves in this region of the bow shock.

The generation and the acceleration mechanism of these different groups of ions have been under intensive investigation for the past four decades, but they are not yet completely understood. A number of mechanisms were proposed for the production of the ion beams. It was demonstrated that ions from the solar wind population can be accelerated to become beam ions if the bow shock is able to turn their velocity direction in such a way to enable them to leave the shock mostly field-aligned in the upstream direction (Sonnerup, 1969). Here it was assumed that the particle energy is preserved in the de Hoffmann-Teller frame. In the de Hoffmann-Teller frame the plasma flow is field-aligned and the electric field is zero. Paschmann et al. (1980) found that the beam peak ion energy agrees well with the predictions of Sonnerup's model as a function of magnetic field orientation relative to the shock normal and solar wind orientation. Burgess (1987) used one-dimensional hybrid simulations combined with the test particle technique to analyze the density of beam ions as a function of shock angle and shock Mach number. He predicted that the density of reflected ions decreases with increasing Mach number and increasing $\Theta_{B n}$.

An alternative mechanism, proposed by Edmiston et al. (1982) and Tanaka et al. (1983), considers the leakage of part of the heated downstream plasma as a mechanism to produce upstream field-aligned beams. Despite the observational evidence by Thomsen et al. (1983), which shows that leakage works in order to produce lower energy beams, the model is not generally accepted. Möbius et al. (2001) demonstrated that field-aligned beam ions originate from the same distribution of ions as the gyrating ions. In a subsequent study, Kucharek et al. (2004) show evidence based on multispacecraft observation that the field-aligned beam ions most probably do not have their origin in the downstream distribution. A recent study based on Geotail observations by Oka et al. (2005) came to the same conclusions. In the past most of the studies regarding the beam ions put the emphasis on their origin and production mechanism, while the temporal and spatial behavior of the field-aligned beam ion population in the upstream region still presents some open questions.

In this paper we will analyze the spatio-temporal evolution of the field-aligned beam ions in front of the Earth's bow shock. As will be demonstrated, the appearance and the characteristics of the beam ions upstream of the quasi-parallel shock is in part the consequence of convection of the magnetic field by the solar wind plasma flow. The study is based on the observation of a high Mach number shock made by Cluster on 18 February 2003. We will present observations which show how the original field-aligned beam ion distribution transforms into an intermediate, and later into a toroidal gyrating ion distribution in the presence of diffuse ions.

The simultaneous observation of the toroidal gyrating and the diffuse ions leads to the conclusion that we deal with two totally different ion populations: while the diffuse ions are coming from the quasi-parallel side of the bow shock, our results show that the toroidal gyrating ions are former field-aligned beam ions and therefore originate on the quasiperpendicular side.

\section{Observation}

\subsection{Energetic ion distributions}

On 18 February 2003, between $\sim 11: 00-23: 00$ UT, Cluster was upstream of the bow shock on an inbound leg of its orbit. This time period was after the passage of a disturbance (most probably a CME) in interplanetary space. A unique characteristics of the event is the absence of the usual level of ultra-low-frequency, large-amplitude fluctuations in the background interplanetary magnetic field. As a result, the magnetic field orientation at all four spacecraft was rather constant, providing connection (i.e., magnetic) to the bow shock over the whole time period. Figure 1 presents the power in the magnetic field versus frequency as obtained from the magnetic field measurements by ACE far from the bow shock and inside the foreshock region as obtained from Cluster magnetic field measurements for the 11:00-11:30 UT time period. The power in the ultra-low frequency waves at ACE is very low and more than two orders of magnitude below the power at Cluster (the spike in the ACE spectrum near $0.08 \mathrm{~Hz}$ is an artifact due to the spacecraft spin). The significant increase of the wave intensity in the foreshock region is the result of the waves which are locally excited by energetic ions which are to be found in the foreshock region. The position of the "peak" in the intensity of waves recorded by Cluster corresponds to the resonant frequency of the FAB ions, as will be discussed later. The characteristics of the monochromatic low frequency waves on this day has been investigated in detail by Archer et al. (2005). We should like to point out that this upstream event is up to now the only one from the Cluster database, which provides us with a continuous, 
almost uninterrupted upstream ion presence for more than $12 \mathrm{~h}$.

The solar wind speed value was rather high, fluctuating slightly around an average value of $\sim 660 \mathrm{~km} / \mathrm{s}$; the Alfvén Mach number $\mathrm{M}_{\mathrm{A}}$ (solar wind speed divided by Alfvén velocity $v_{A}$ ) was $\sim 8$. Figure 2 presents from top to bottom the spacecraft trajectory and the bow shock in the $x-y$ and $x-z$ planes of the GSE (Geocentric Solar Ecliptic) coordinate system; the solar wind bulk velocity, the interplanetary magnetic field components and the solar wind plasma density versus time for the time period under investigation. The solar wind density and bulk velocity data were obtained from the CISHIA instrument (Rème et al., 2001), which covers the ion energy range up to $32 \mathrm{keV}$; the magnetic field components were provided by the FGM instrument (Balogh et al., 2001). The data from the CIS-HIA and FGM have a 1 spin (i.e. 4 s) resolution. In order to filter out the high frequency fluctuations in the magnetic field and to decrease the statistical error in the ion data we averaged these data over 8 spins (i.e. $32 \mathrm{~s}$ ). The location of the bow shock is calculated by using a dynamic bow shock model (Peredo et al., 1995): the exact location of the bow shock is known from the observed crossing by the spacecraft at $\sim 22: 40$ UT. Using the solar wind parameters at the time of the crossing as a priori information, the shape and the position of the bow shock is then dynamically modified according to the actual observed solar wind parameters (i.e. solar wind plasma density and bulk velocity) for the time period under investigation. The bow shock position shown in Fig. 2 corresponds approximately to the average position of the bow shock for the time period under investigation. In the bottom panel of Fig. 2 are indicated by horizontal bars the time periods for which we will analyze in detail the ion distribution.

Kis et al. (2004) analyzed the diffuse ion behavior during this time period in the $10-32 \mathrm{keV}$ energy range. Based on the e-folding distance of the intensity they have concluded that the diffuse ions are subject to diffusive transport in the upstream region and obtained a mean free path for $32 \mathrm{keV}$ ions of $\sim 2.4 R_{E}$. In the present study we extend the analysis to lower energies in order to study the behavior of the field-aligned beam ions in the foreshock region. We focus on the protons, since they are the main component of the solar wind as well as of the energetic ion population. For this study we use the magnetic field data provided by the FGM instrument and the ion data provided by the CODIF analyser of the CIS instrument (Rème et al., 2001). The CODIF analyzer covers the ion energy up to $40 \mathrm{keV}$. The reason that in the present paper data from the CODIF analyzer is used is that this instrument is capable to distinguish protons from other ions. We perform the analysis over the time period between 11:00-14:00 UT. We will use only FGM and CODIF data from spacecraft 1 (SC1) since this spacecraft has the highest sensitivity ion data during the time period of observations; data from the other three spacecraft are unreliable for this time period due to either technical problems and/or sensi-

\section{1:0011:30 UT, February 18, 2003}

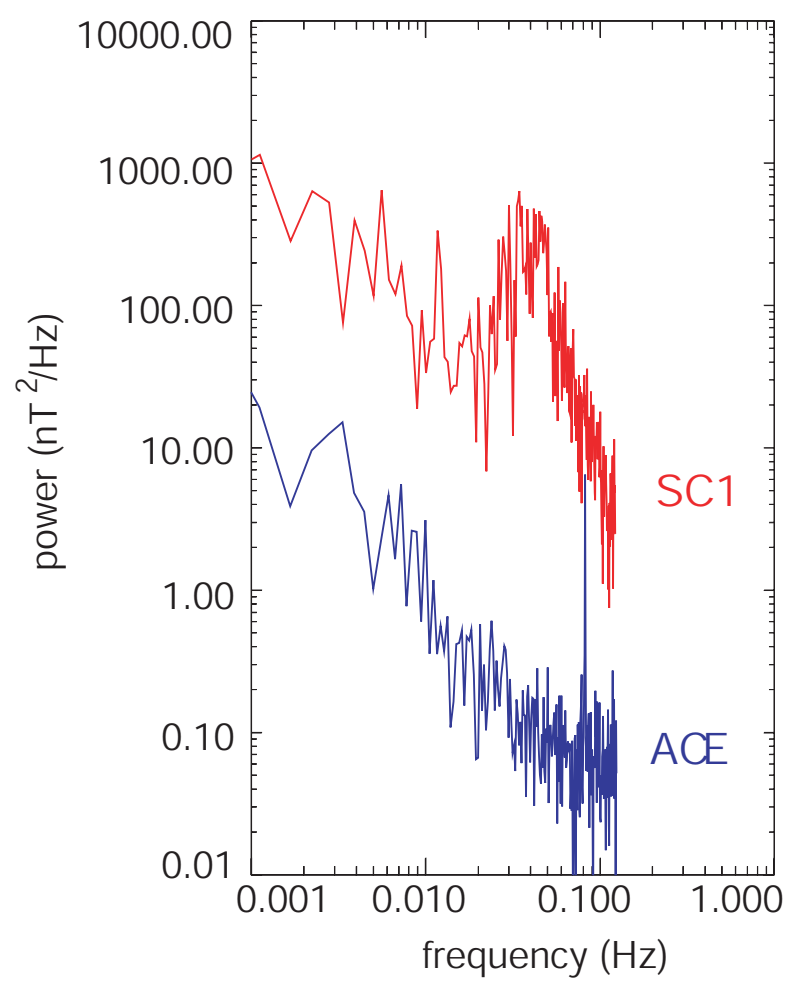

Fig. 1. Magnetic wave intensity versus frequency in the interplanetary region (recorded by ACE) and inside the foreshock region (recorded by Cluster) for the time period of 11:00-11:30 UT. ACE magnetic field data courtesy of C. W. Smith.

tivity presets of the instruments. Figures 3-4 and Figs. 6-8 show the particle distributions at different times in $v_{\|}$(velocity component parallel to the magnetic field) versus $v_{\perp}$ (velocity component perpendicular to the magnetic field) velocity space. This is a cut of the full 3-D distribution in the plane defined by the solar wind bulk velocity vector and the average magnetic field vector. The solar wind distribution appears in the figure as an elongated, red-colored patch; this is due to the fact that the CODIF sensor, to avoid saturation and degradation in the high gain section, does not sweep through the whole solar wind distribution, but stops at $\sim 2 \mathrm{keV}$ in a $45^{\circ}$ wide sector centered in the solar wind direction. Because of the limited sweep the solar wind distribution is incomplete, and the color does not represent the real value of the solar wind flux (i.e., approximately 5 percent of the total solar wind ion flux value is presented). In all other areas of velocity space outside of the red patch the color coding represents the physical value of the ion flux. The offset of the solar wind in the $v_{\perp}-v_{\|}$plot from $v_{\perp}=0$ is due to the relative angle between the average magnetic field and the solar wind bulk velocity. The distributions are obtained by averaging over $300 \mathrm{~s}$. This averaging has been performed firstly 


\section{February, 2003}
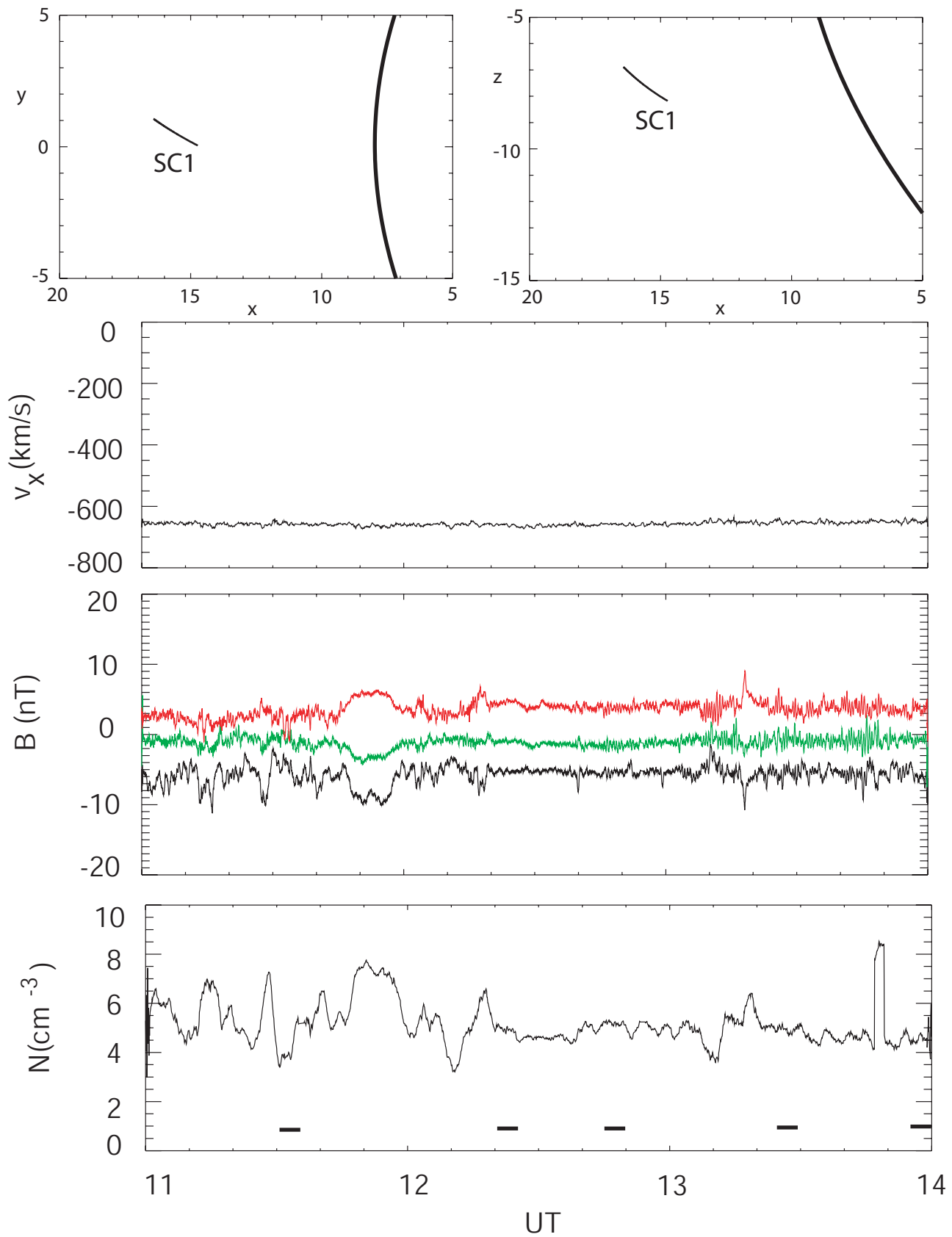

Fig. 2. From top to bottom: spacecraft (SC1) trajectory and bow shock location in the $y-x$ (left) plane and in the $z-x$ (right) plane, respectively, solar wind bulk velocity parallel to $x_{\mathrm{gse}}$, interplanetary magnetic field components and solar wind plasma density versus time for the time period under investigation. Horizontal bars in the bottom panel indicate the time periods for which ion distributions are analyzed in detail.

in order to obtain the distribution with sufficient statistical accuracy over a wide velocity range and second in order to smooth out fluctuations due to short time directional fluctuations of the magnetic field. In the panel below the velocity distribution we present the magnetic field vector components in the GSE coordinate system versus time for a $10 \mathrm{~min}$ time period which contains the time period of the velocity distribution.

At 11:30-11:35 UT the spacecraft (in the following all references in this chapter to "spacecraft" will refer to SC1) was situated in the upstream region and was connected along the magnetic field to the quasi-parallel bow shock. The 

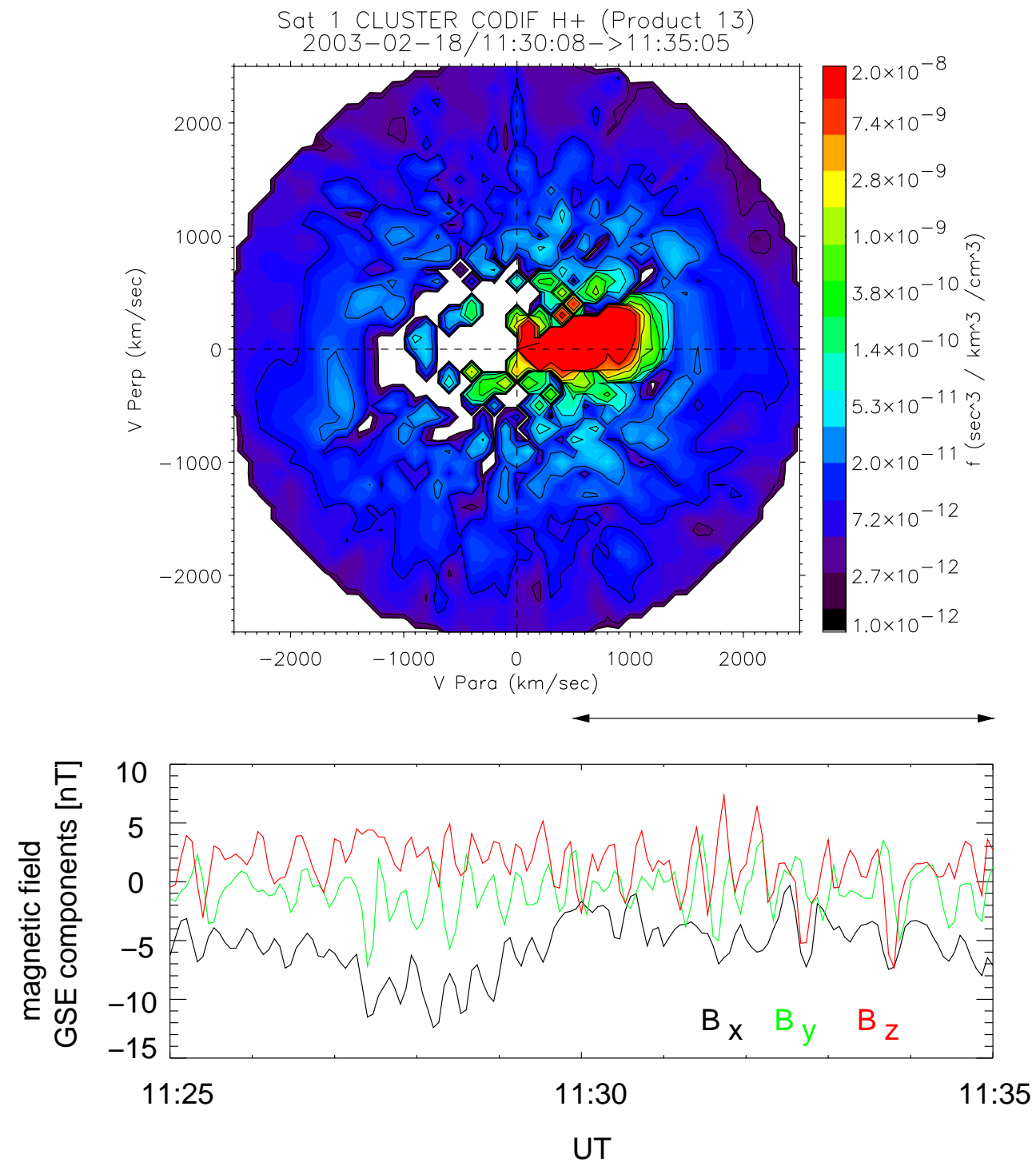

Fig. 3. The upper part presents the proton distribution in velocity space observed by SC1 CIS-CODIF during the time period 11:3011:35 UT. The lower shows the associated interplanetary magnetic field vector components in the GSE coordinate system during the same time period.

distribution at 11:30-11:35 UT, presented in Fig. 3, shows the cut of a broad, shell-like ion distribution. It was demonstrated by Paschmann et al. (1981) that this kind of distribution is a characteristic diffuse ion distribution. The lower panel shows low-frequency, high-amplitude magnetic fluctuations at the same time; these fluctuations can always be observed in the presence of diffuse ions (Hoppe et al., 1981). It has been shown by Eastwood et al. (2005) that the magnetic waves propagate in the upstream direction along the magnetic field with a velocity of $\sim v_{A}$, but since the speed of wave propagation is substantially lower than the solar wind bulk velocity, these waves are convected to the shock by the plasma flow. This also means that the waves are generated in-situ, i.e. they cannot come from the downstream direction.
Figure 4 shows the ion distribution and the associated magnetic field for the time period between 12:20-12:25 UT. At this time a field-aligned beam is observed. On the left side of the velocity space plot the FAB can be identified as a localized, high flux of protons directed into the upstream direction along the magnetic field streaming against the incoming solar wind. The FAB is a beam of solar wind ions which are reflected at the quasi-perpendicular side of the bow shock and which have a velocity of about twice the solar wind bulk speed in the solar wind frame (e.g., Eastwood et al., 2005). It has been shown by Paschmann et al. (1979) and recently by Eastwood et al. (2005) that at the location where a FAB is observed usually there are either no fluctuations at all or very low fluctuation levels in the magnetic field. This fact can 

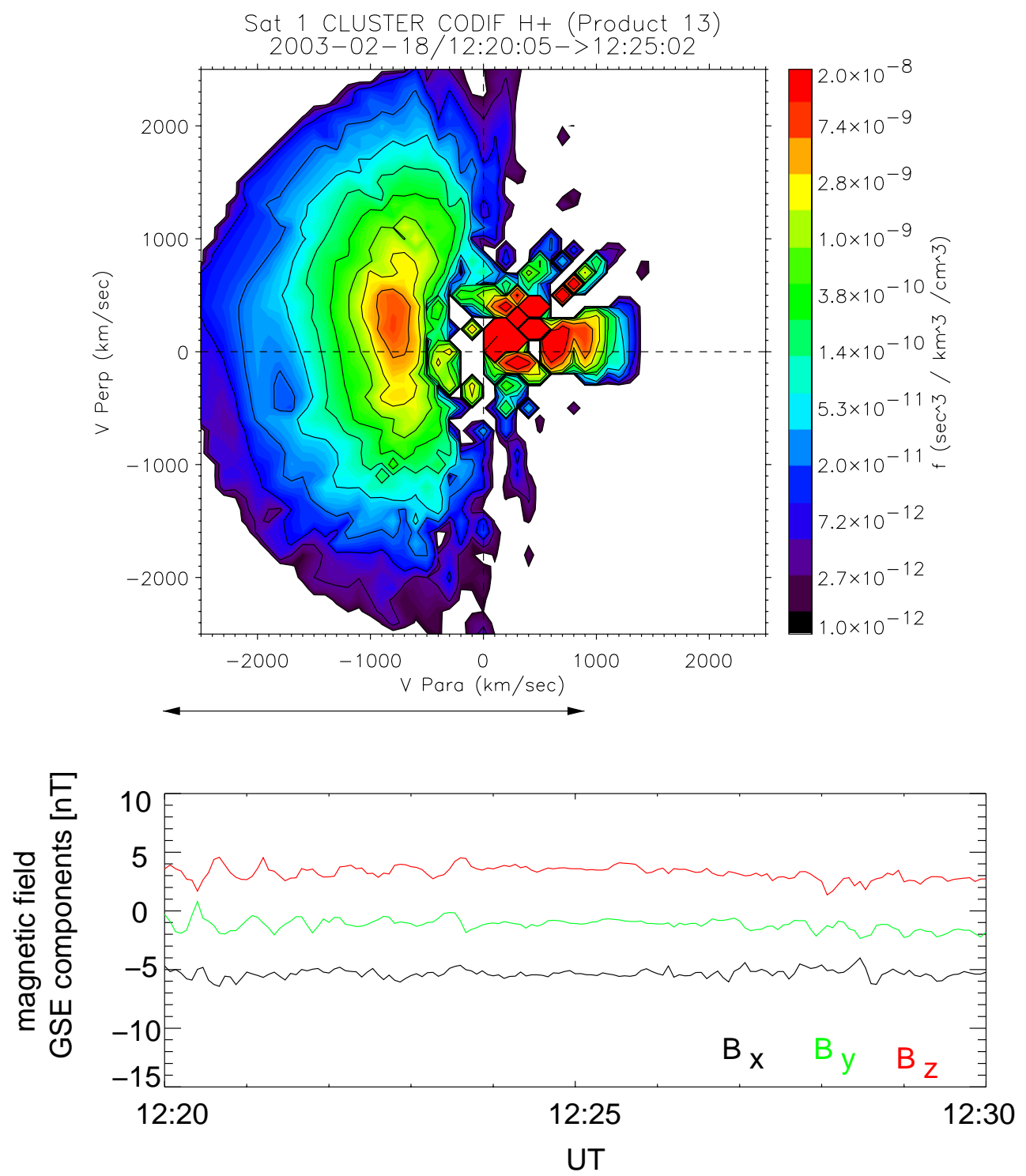

Fig. 4. Same as Fig. 3 for the time period 12:20-12:25 UT.

be seen in the lower panel, where the magnetic field components do indeed not exhibit large fluctuations. The velocity distribution plot shows that ions backstreaming towards the shock are almost absent. In addition to the FAB ions are higher velocity ions streaming away from the shock. These ions have velocities reaching above $2000 \mathrm{~km} / \mathrm{s}$ in the spacecraft frame. Figure 5 shows a cut of the distribution function along $v_{\|}$, the velocity parallel to the magnetic field. Negative velocities are in the sunward direction, positive velocities are in the direction of the bow shock. The solid line shows the distribution averaged over the 12:20-12:25 UT time interval and clearly exhibits the field-aligned beam. In addition, at velocities above $|-1800| \mathrm{km} / \mathrm{s}$ (i.e., on the $v_{\|}$negative side) a high energy tail can be observed as a second population, corresponding to the blue region in the distribution function of Fig. 4. The dashed line in Fig. 5 shows the distribution function of diffuse upstream ions obtained between 11:30$11: 35$ UT. The large increase between $-100 \mathrm{~km} / \mathrm{s}$ and $0 \mathrm{~km} / \mathrm{s}$ is due to the 1 count limit; the increase below $1000 \mathrm{~km} / \mathrm{s}$ is due to the unresolved solar wind. As can be seen the phase space density in the high energy tail of the beam distribution corresponds to the phase space density of the diffuse ions.

The distribution in Fig. 6 presents the particle distribution for the time period between 12:45-12:50 UT. In the lower panel we can observe a strong wave activity at this time. These waves scatter the particles in pitch angle, the originally focused beam ion population now presents a kidney-shaped, i.e., intermediate distribution. Beside this intermediate distribution of lower energy ions there is superposed an almost complete shell-like distribution of higher-energy diffuse ions. 


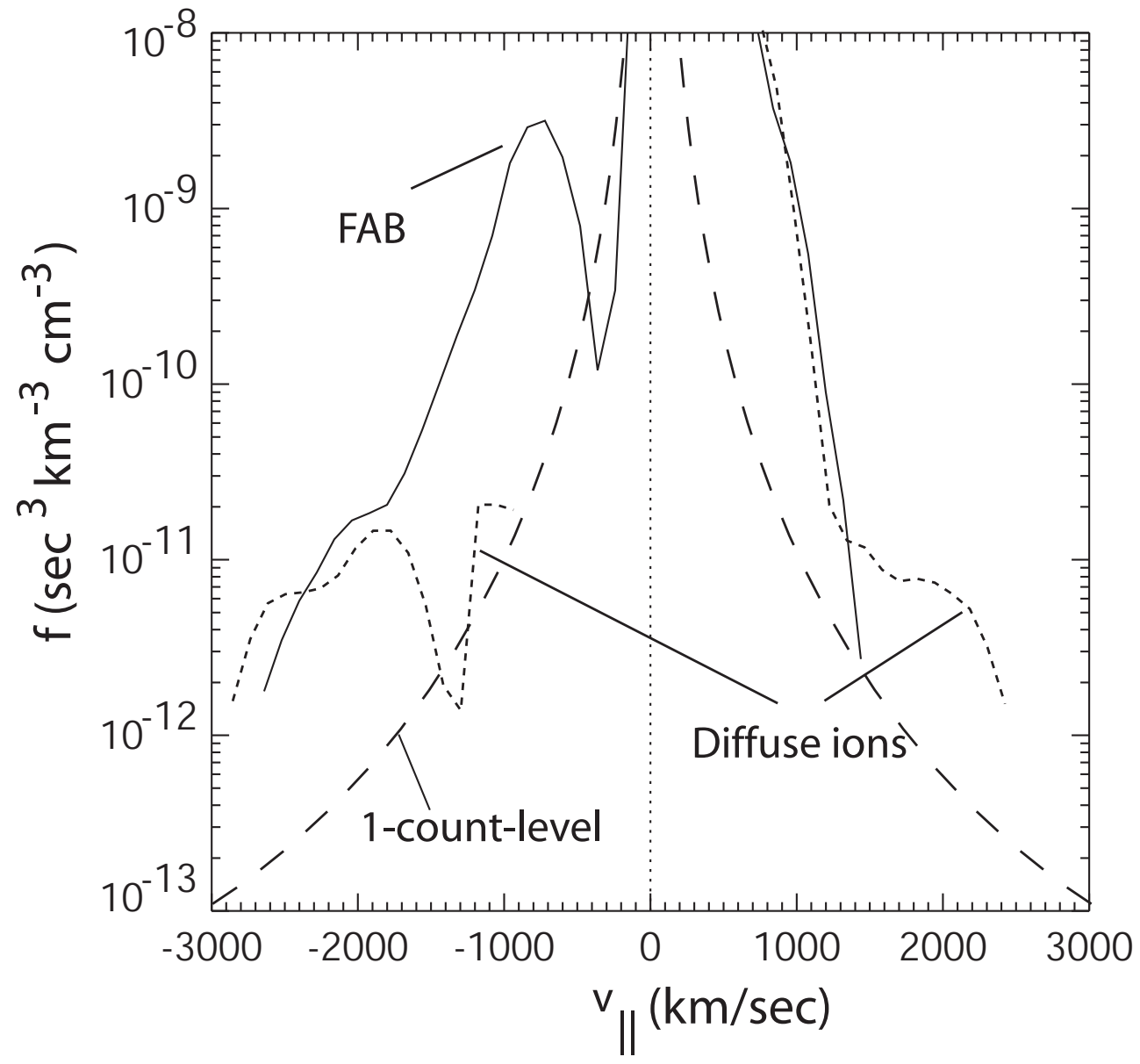

Fig. 5. Phase space density along a cut in velocity parallel to the magnetic field. Solid line: Distribution averaged over time period 12:20-12:25 UT, dashed line: Distribution averaged over time period 11:30-11:35 UT. Also shown is the one-count level.

As a result of the wave activity the intermediate ions are also partially backscattered and move toward the shock (in the spacecraft frame). The distribution in Fig. 7 shows the particle distribution at 13:25-13:30 UT in a more evolved phase of the scattering: the diffuse ion shell is already closed and a substantial fraction of ions forming the kidney-shaped part of the lower-energy ion distribution are backscattered. Finally, Fig. 8 shows the distribution at 13:55-14:00 UT: the diffuse ions represent a higly isotropic, broad, closed shell-like distribution, similar to the distribution observed at 11:3011:35 UT. The original FAB ions, which earlier formed a kidney-shaped (i.e. intermediate) distribution, at this time present a toroidal gyrating ion distribution: ions with small pitch-angle values are missing. We will argue that this is due to the combination of pitch-angle scattering and convection by the solar wind: ions with a larger upstream directed parallel velocity (i.e. with smaller pitch-angle) have already escaped in the upstream direction, while the ions with pitch angles closer to $90^{\circ}$ are convected with the magnetic field deeper into the foreshock by the solar wind plasma flow. In the following we present a more detailed discussion of the observed energetic ion distributions and the mechanism which leads to these distributions.

\subsection{Relation to bow shock geometry}

The upstream event observed by SC1 between 11:0014:00 UT on 18 February 2003, provides the possibility to analyze in detail the spatio-temporal evolution of the FAB ion population in front of the Earth's quasi-parallel bow shock. This possibility is the result of the combination of several key conditions, like the absence of ultra-low frequency, large-amplitude fluctuations in the far upstream interplanetary magnetic field (which results in the unusual stability of the interplanetary magnetic field direction over a time period of many hours), the high solar wind velocity, and the favorable position of the spacecraft. It can be observed how the distribution of the FAB transforms first into an intermediate, and after that into a toroidal gyrating ion distribution while it is convected by the solar wind plasma flow deeper into the foreshock region. 

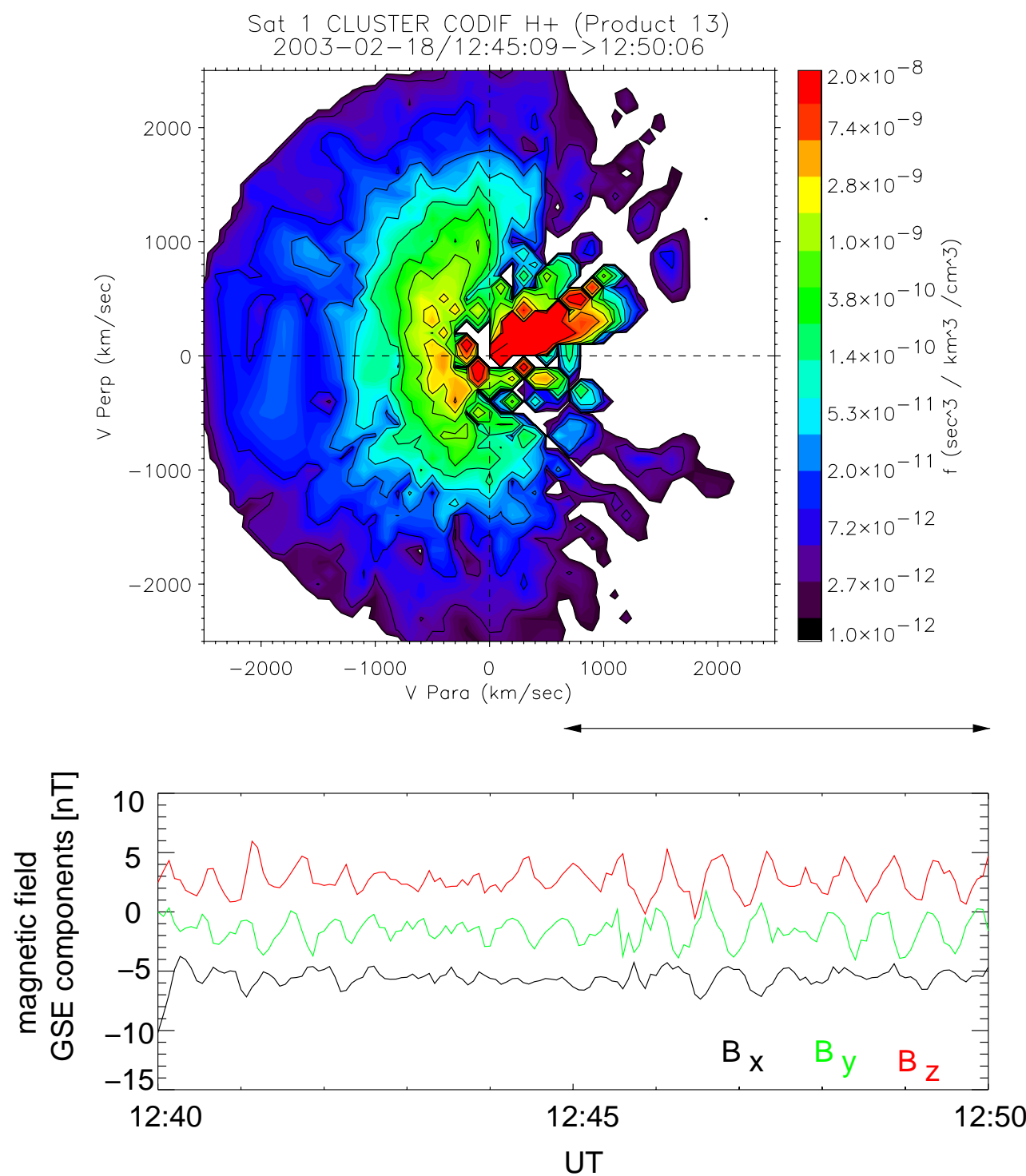

Fig. 6. Same as Fig. 2 for the time period 12:45-12:50 UT.

In order to understand the physical mechanism which leads to the outlined transformation of the original FAB distribution, we need to know the location of the spacecraft and the direction of the magnetic field related to the bow shock surface. Figure 9 presents from top to bottom the evolution of the spacecraft distance to the shock along the magnetic field, the angle between the interplanetary magnetic field and $x_{\mathrm{GSE}}$, and $\Theta_{B n}$ versus time for the 11:00-14:00 UT time period. The distance of the spacecraft to the shock along the magnetic field line (upper panel) and $\Theta_{B n}$ (lower panel) were calculated by using a dynamical bow shock model, as described above. Between 11:30 and 11:35 UT the ion instrument of the spacecraft observes a broad shell-like shaped, diffuse energetic ion distribution in the presence of high-amplitude, low-frequency fluctuations in the magnetic field. At this time the spacecraft is located in the foreshock region and magnetically connected to the quasi-parallel side of the bow shock, $\Theta_{B n}$ being $\sim 14^{\circ}$. The distance to the shock along the magnetic field is $\sim 7.7 R_{E}$. The ion distribution in velocity space has a hole in the center which means that the intensity of the lower-energy ions in this region is very small, i.e. its value falls below the one count limit of the instrument.

For the time period under investigation the value of $B_{y}$ (interplanetary magnetic field vector component parallel to $\left.y_{\mathrm{GSE}}\right)$ is substantially smaller than the value of $B_{x}$ or $B_{z}$. For most of the time $B_{y}$ fluctuates slightly around the zero value. Because of this the changes in the magnetic field direction are mainly due to changes in the values of $B_{x}$ and $B_{z}$. The temporal evolution of the angle $\alpha$ between the $x_{\mathrm{GSE}}$ and the interplanetary magnetic field thus provides information 

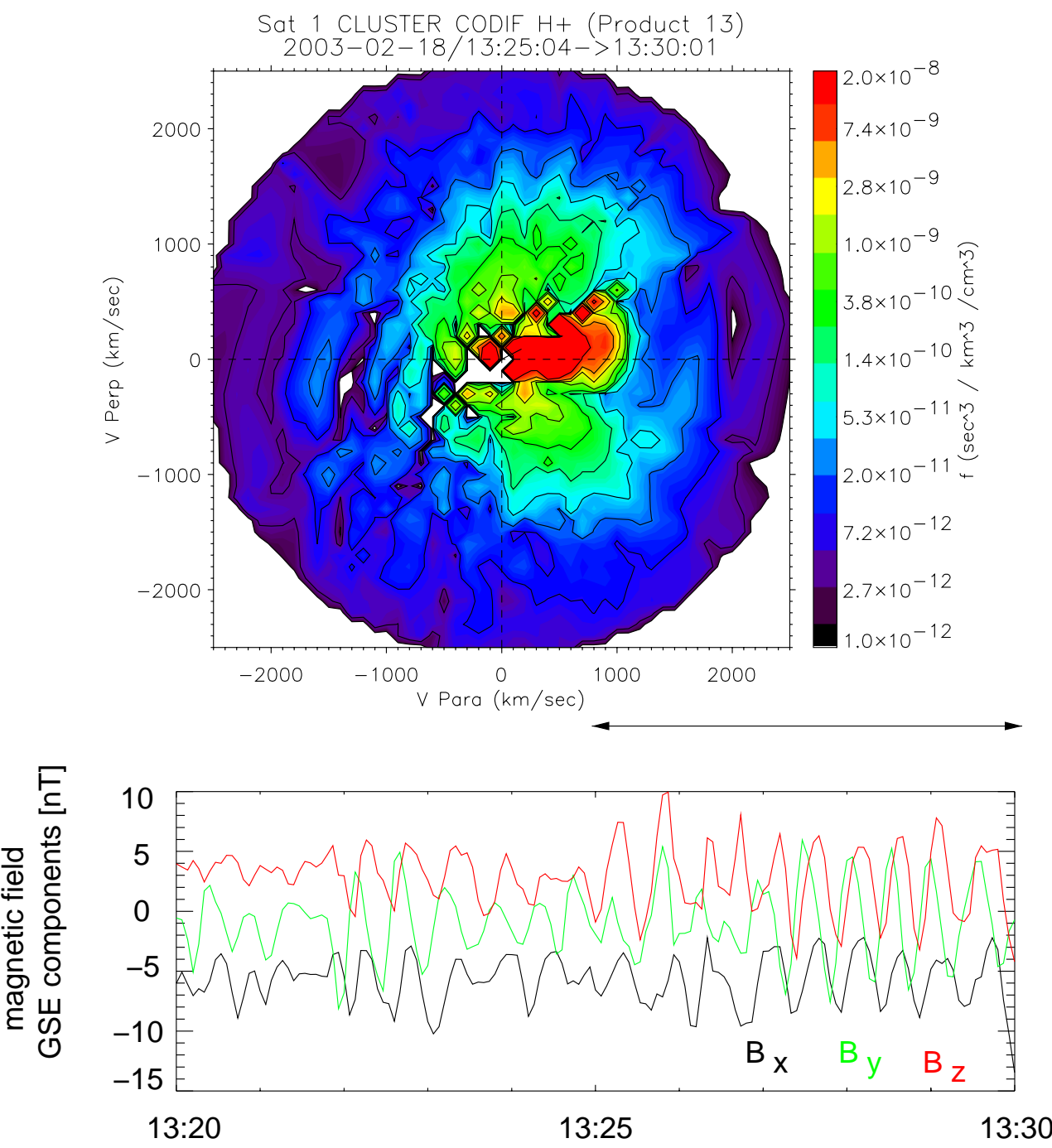

Fig. 7. Same as Fig. 2 for the time period 13:25-13:30 UT.

about the foreshock geometrical structure and the ion foreshock boundary and helps to understand the physical process. As it can be seen from Fig. 9, middle panel, at 11:30 UT $\alpha$ is $\sim 15^{\circ}$.

Later the magnetic field direction changes and the spacecraft is at $\sim 12: 25 \mathrm{UT}$ able to observe the field-aligned beam (FAB). At this time the spacecraft distance to the bow shock is $\sim 7.5 R_{E}, \alpha$ is $\sim 30^{\circ}$, and $\Theta_{B n}$ is $\sim 25^{\circ}$. The associated fluctuations in the magnetic field are small. It has been demonstrated earlier by Greenstadt et al. (1980) and Thomsen (1985) that a FAB can be observed in a narrow region near the ion foreshock boundary. The FAB ions are thought to be solar wind ions reflected at the quasi-perpendicular side of the bow shock. After their reflection these ions travel in the upstream direction along the magnetic field with a velocity of about twice the solar wind bulk velocity (in the plasma frame). The reflection occurs as a result of the "head-on" collision of the solar wind ions with the shock wave. As can be seen from Figs. 4 and 5 the peak value of the beam flux in the velocity space is located at $v_{\|} \sim-750 \mathrm{~km} / \mathrm{s}$ and $v_{\perp} \sim 500 \mathrm{~km} / \mathrm{s}$. This results in a total beam ion velocity of $\sim 900 \mathrm{~km} / \mathrm{s}$ in the spacecraft frame. Since the magnetic field is "frozen-in" in the solar wind the magnetic field is convected by the plasma flow. Therefore a spacecraft situated in the upstream region is able to observe a particle velocity which is the resultant velocity of the particle movement along the magnetic field and the convection of the field by the plasma. By taking into account the particle velocity along the magnetic field and the plasma convection the trajectory of the FAB can be determined. The direction of the magnetic field is known at the time of the observation as well as the velocity and the direction of the plasma convection, the latter is in fact the solar wind bulk velocity. Using these data we computed the ion beam velocity along the interplanetary magnetic field 

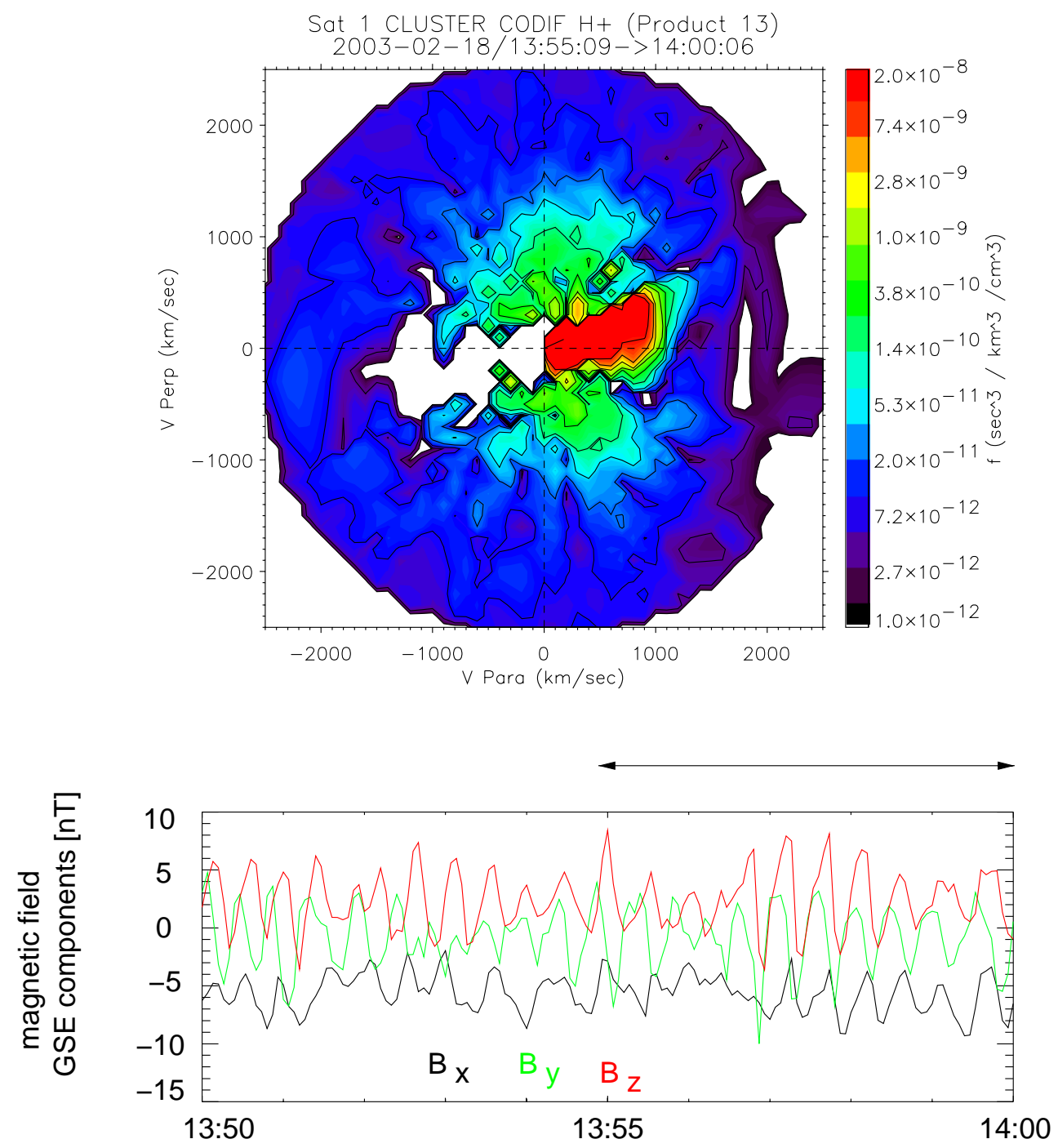

Fig. 8. Same as Fig. 3 for the time period 13:55-14:00 UT.

line. Results show that the beam observed at $\sim 12: 25$ UT by the $\mathrm{SC} 1$ is an ion beam which consists of ions which propagate along the magnetic field with a velocity of $1300 \mathrm{~km} / \mathrm{s}$ in the plasma frame. Since the solar wind velocity at this time is $\sim 650 \mathrm{~km} / \mathrm{s}$ (i.e. half the beam velocity) we conclude that the FAB ions have twice the velocity of the solar wind ions. This is in good agreement with recent results of Eastwood et al. (2005), who performed a statistical analysis of a number of events based on Cluster observations and found that in the solar wind rest frame $v_{b} / v_{s w}=2.1 \pm 0.6$, where $v_{b}$ and $v_{s w}$ are the beam and solar wind velocity, respectively.

Taking into consideration the direction of the interplanetary magnetic field, the solar wind and the beam velocities and the position and shape of the bow shock, we have calculated the trajectory and the point of origin of the FAB on the bow shock surface. The results show that the FAB originates on the quasi-perpendicular side of the bow shock at a posi- tion where the $\Theta_{B n}$ is $\sim 60^{\circ}$. This is also in good agreement with earlier observations.

In addition to the beam ions in the distribution shown in Fig. 4 one can see the presence of $\sim 30 \mathrm{keV}$ ions (i.e. ions with velocities higher than $\sim 2400 \mathrm{~km} / \mathrm{s}$ ). The geometrical configuration does not allow the spacecraft to be reached by $30 \mathrm{keV}$ energy ions originating at the quasi-perpendicular side of the shock. Instead, the $30 \mathrm{keV}$ protons have to come from the quasi-parallel side of the bow shock, as will be discussed below.

The important feature of this situation is that the spacecraft is able to observe particles coming from the quasi-parallel and the quasi-perpendicular side of the shock simultaneously, or in other words it can observe the field-aligned beam ions and higher energy, quasi-parallel shock-accelerated ions at the same time (note that the spacecraft is connected along the magnetic field to the quasi-parallel side of the shock, $\Theta_{B n}$ 

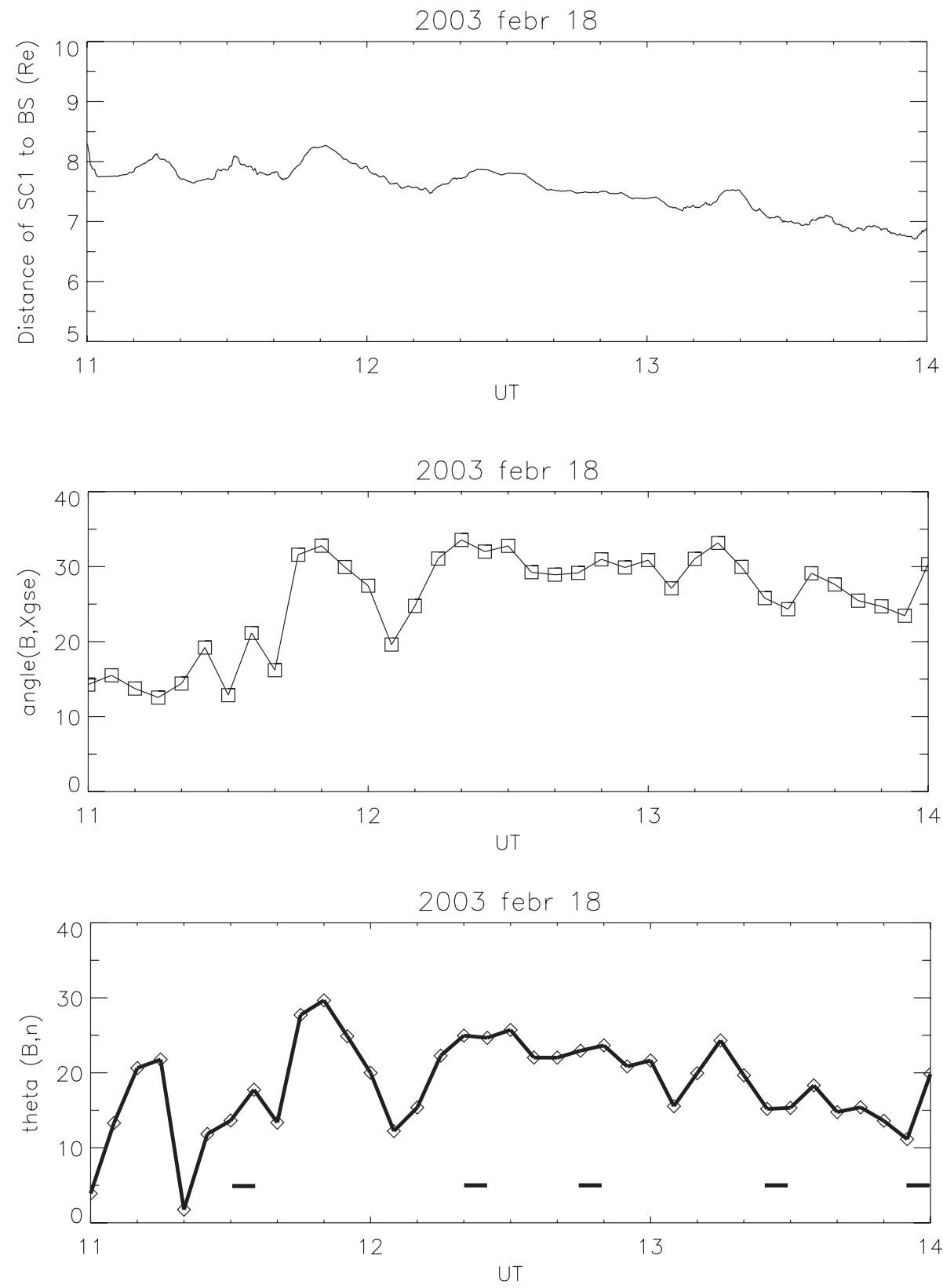

Fig. 9. From top to bottom: evolution of the distance of SC1 to the bow shock along the magnetic field, the angle between the interplanetary magnetic field and $x_{\mathrm{GSE}}$, and the shock normal - magnetic field angle $\theta_{B n}$ versus time for the time period under investigation. Horizontal bars in the bottom panel indicate the time periods for which ion distributions are analyzed in detail.

being $\sim 25^{\circ}$ ). This results in a superposition of the different ion populations and explains why besides the FAB ions there are also higher-energy ions present in the distribution.

At $\sim 12: 45-12: 50$ UT the spacecraft is situated deeper in the foreshock region due to a change in the interplanetary magnetic field direction (see Fig. 6). It can be seen that in this region there are small amplitude waves. These waves scatter the FAB ions in pitch-angle around a circle with a center point depicted by the solar wind beam position minus the $v_{A}$ since the ion energy is preserved in the wave frame. The distribution shows that the originally concentrated ion beam now has a more kidney-shaped distribution meaning that some of the beam ions started to acquire larger pitch angle values due to the interaction with the magnetic waves. In addition it can be observed that the former higher-energy ion half-shell started to form a shell, however the shell is not yet complete at this position. At a later time period, between 13:25-13:30 UT, the spacecraft is situated even deeper 
13:20 - 13:30 UT, February 18, 2003

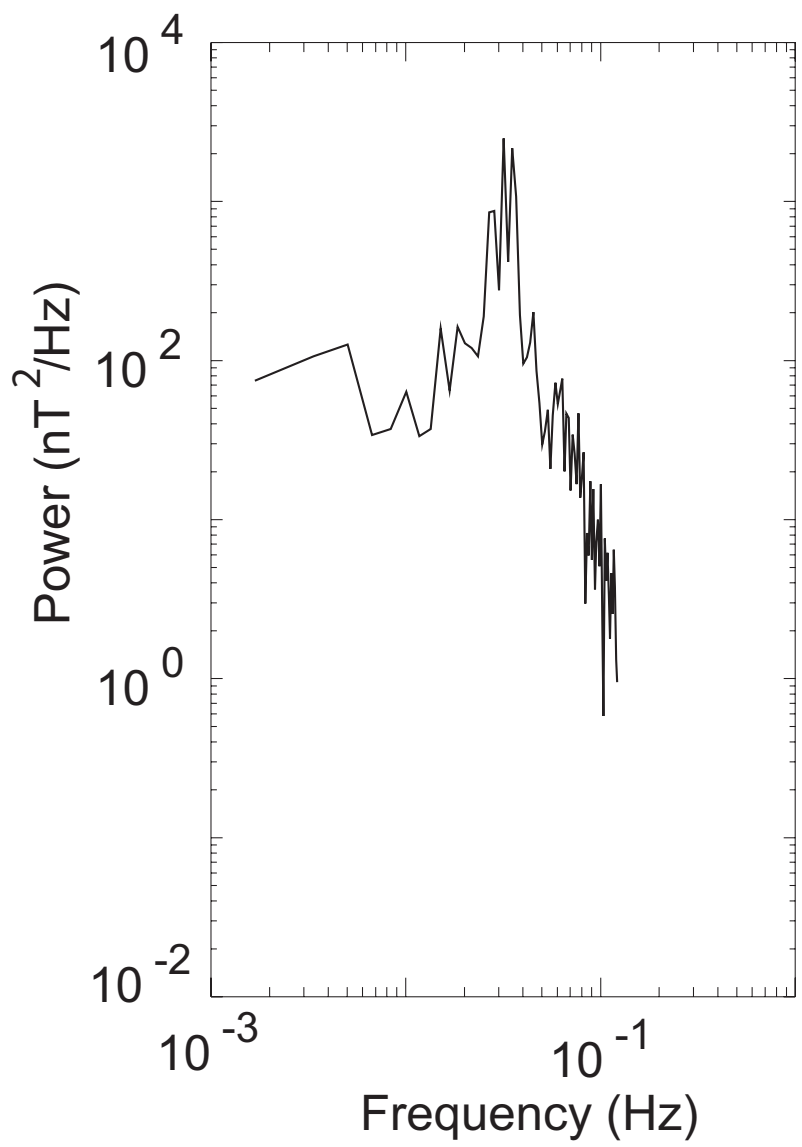

Fig. 10. Magnetic field power versus frequency for the 13:2013:30 UT time period.

in the foreshock region than at 12:45-12:50 UT due to its inbound orbit and due to a further slight change in the interplanetary magnetic field direction. Large-amplitude low frequency waves can be observed in Fig. 7; these waves efficiently scatter the energetic particles in pitch-angle. The diffuse ion distribution is a closed, broad shell-like distribution showing that a substantial amount of high-energy ions are backscattered. The lower energy former FAB ions still have a kidney-shaped distribution, but compared to the distribution observed at 12:45-12:50 UT this distribution has a more elongated kidney-shape, similar to a half-shell. This shows that at this time/position part of the ions are gyrating (i.e. have large pitch angles) with positive parallel velocity oriented towards the shock. In velocity space the intensity of ions having pitch angles close to zero (i.e. maximum parallel velocity in the upstream direction) is very low. Evidently, this is a result of the pitch-angle scattering process combined with convection from the solar wind. The convection results in a velocity filter effect spatially separating ions having the same energy but different pitch angles. Ions with maximum parallel velocity (i.e. with pitch angles close to zero) oriented upstream along the magnetic field lines are moving faster along the magnetic field than the ions which have larger pitch angles, while they all are convected by the solar wind plasma flow. The hole in the center of the kidneyshaped distribution is the result of the spatial separation of ions with different pitch angles.

Figure 10 presents the total wave power versus frequency during 13:20-13:30 UT. During this time period the average magnetic field magnitude was $\sim 7.61 \mathrm{nT}$, the solar wind velocity was $\sim 650 \mathrm{~km} / \mathrm{s}$ and the plasma number density was $\sim 4.9 \mathrm{~cm}^{-3}$. Using these values we obtain $\sim 74 \mathrm{~km} / \mathrm{s}$ for the Alfvén speed and $\sim 0.11 \mathrm{~Hz}$ for the proton cyclotron frequency. Taking into account the average direction of the interplanetary magnetic field relative to the solar wind bulk velocity $\left(\sim 33^{\circ}\right)$ and assuming parallel wave propagation one obtains for the resonant frequency of FAB ions with a speed of twice the solar wind speed a value of $\sim 0.039 \mathrm{~Hz}$ in the spacecraft frame. As can be seen from Fig. 10 this resonance frequency of the FAB ions is close to the location of the peak in the wave power. This fact demonstrates that the FAB ions will be scattered by self-induced waves. We calculated also the resonant frequency for the $30 \mathrm{keV}$ (i.e. diffuse) ions assuming maximum parallel velocity and obtain a value of $\sim 0.022 \mathrm{~Hz}$. Thus diffuse ions are most probably not efficiently scattered by the waves excited by the FAB. The diffuse ions are most probably scattered by the waves at lower frequencies (below the peak in Fig. 10) which are self-excited waves. This is strictly only true for diffuse ions with velocities parallel to the field; at pitch angles closer to $90^{\circ}$ the parallel velocity is smaller and the resonance can be at higher frequencies.

The final phase of this process can be observed at 13:5514:00 UT (Fig. 8) where it can be seen that only toroidal gyrating lower-energy ions are present at this position in the foreshock region. As can be seen from the large hole in the center of the distribution all former FAB ions with, after pitch angle scattering, small pitch angles are now missing. The higher-energy diffuse ions present a broad, shell-like distribution. At this time the spacecraft distance to the bow shock is $\sim 6.7 R_{E}$, and $\Theta_{B n}$ at the intersection point of the magnetic field line through the spacecraft with the bow shock is $\sim 15^{\circ}$.

\section{Discussion}

At 11:30-11:35 UT the distance of the spacecraft to the bow shock was $\sim 7.5 R_{E}$ and $\Theta_{B n} \sim 15^{\circ}$, at 13:55 UT the distance was $\sim 6.7 R_{E}$ and $\Theta_{B n}$ was $\sim 15^{\circ}$. Despite similarity of $\Theta_{B n}$ and of the distance at the two different times we observe a large difference in the velocity-space ion distributions. If the lower-energy, gyrating ions observed in the distribution at 13:55 UT would come directly from the shock, we should be able to observe them also at 11:30 UT, due to the very close values in distance to the bow shock and $\Theta_{B n}$. The fact 

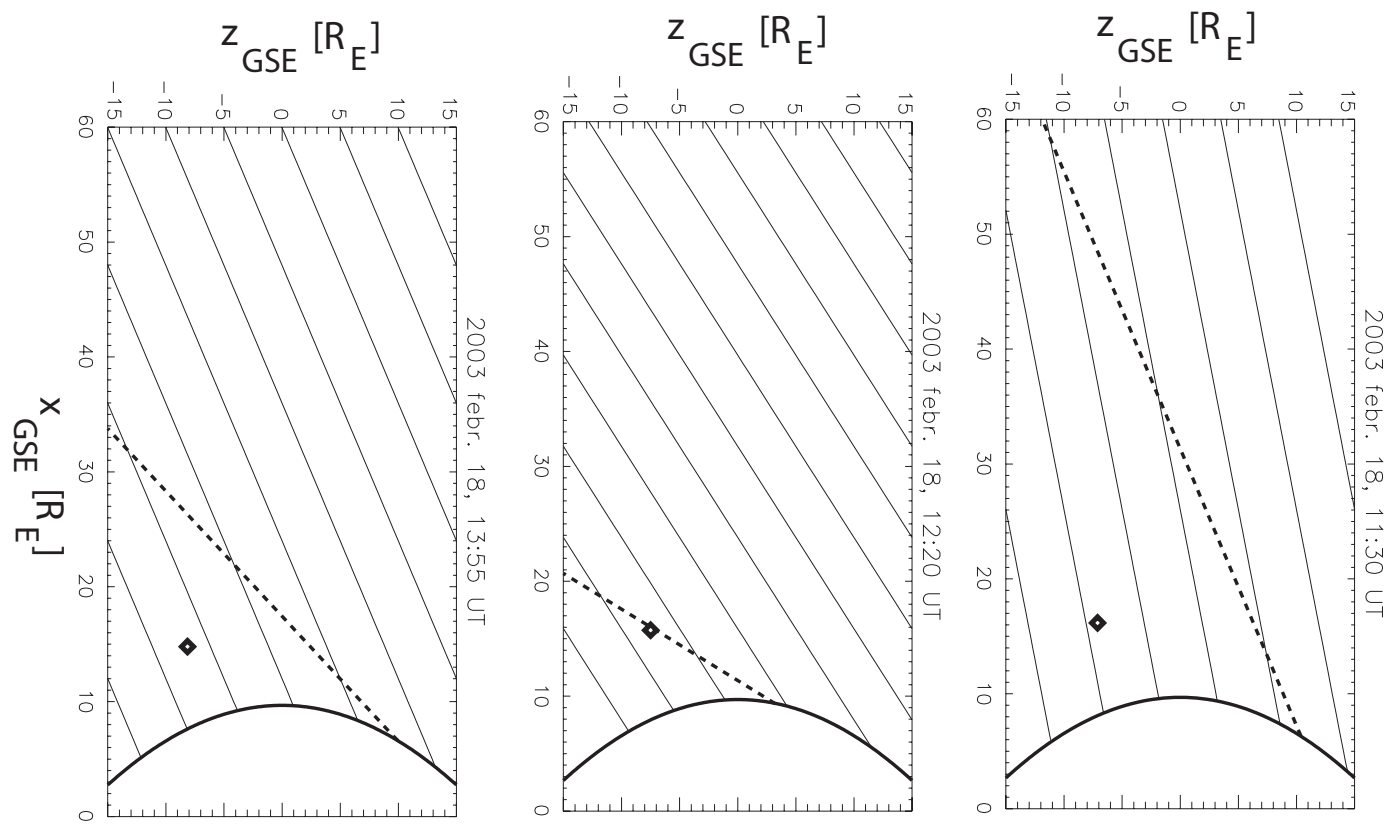

Fig. 11. The three figures present the bow shock position (curved line), the location of the spacecraft (diamond-shaped symbol) and the FAB (dashed line) in the $x_{\mathrm{GSE}}-z_{\mathrm{GSE}}$ plane at 11:30, 12:20 and 13:55 UT. Also depicted are the projection of the interplanetary magnetic field direction in the plane of representation.

that the toroidal gyrating ions are missing from the distribution observed at 11:30 UT, shows that these ions are indeed the remnants of the original FAB. In our interpretation the FAB is pitch-angle scattered by the low frequency upstream waves and due to the described velocity-filter effect they are separated according to their pitch angle, i.e. their velocity component parallel to the magnetic field, which leads deeper in the foreshock region to the presence of a lower-energy, toroidal gyrating ion distribution. This latter distribution is superposed to the higher-energy, shell-like diffuse ion distribution. Also the fact that the whole process takes place at the distance interval 7.5-6.7 $R_{E}$ from the bow shock suggests that the distance from the shock is not the important factor, but rather the distance of the spacecraft from the FAB, or in other words, the spacecraft distance from the ion foreshock boundary. To show this, we reconstructed the position of the FAB at 11:30, 12:20 and 13:55 UT. For the reconstruction we used the bow shock model and we have taken into account the solar wind and FAB velocity, and the direction of the interplanetary magnetic field. Figure 11 shows from top to bottom, in the $x_{\mathrm{GSE}}-z_{\mathrm{GSE}}$ plane, the location of the bow shock, spacecraft and FAB at 11:30,12:20 and 13:55 UT. The point of origin of the FAB for the times at 11:30 and 13:55 UT has been calculated by assuming that the beam originates on the quasi-perpendicular side of the bow shock, where $\Theta_{B n}$ is $60^{\circ}$. The direction of the interplanetary magnetic field has also been taken into account. It can be seen from Fig. 11 that at 13:55 UT the spacecraft is situated at a distance of $\sim 11 R_{E}$ from the FAB (dashed line) in the downstream di- rection. Since at this time the spacecraft observes a toroidal gyrating distribution of the former FAB ions, this shows, at least in the case of this study, that the distance of $\sim 11 R_{E}$ from the FAB is the distance over which the FAB ions are efficiently scattered and spatially separated according to their pitch angle, leading to the presence of only toroidal gyrating ions at the spacecraft location. Figure 11 also demonstrates that the reason why we are not able to observe a toroidal gyrating component at 11:30 UT is the fact that at this time the spacecraft is situated at a distance of $\sim 31 R_{E}$ from the FAB. Obviously, over this distance the former FAB ions are so efficiently scattered and separated due to the velocity filter effect that at the spacecraft location their intensity is so low, that they cannot be detected anymore.

The scattered and backstreaming ions of the FAB interact eventually with the bow shock in the more quasi-parallel region and can take part in a diffusive acceleration process. FAB ions can thus be a source for diffuse ions. On the other hand it is well documented that the quasi-parallel bow shock itself injects ions into a diffusive acceleration process. Ipavich et al. (1988) have actually reported that the percentage of alpha particles in the FAB is rather low, while the alpha to proton ratio in diffuse ions (at the same energy per charge) corresponds well to the same ratio in the solar wind (Ipavich et al., 1984) and have excluded on the basis of these observations the FAB as a major source for diffuse ions. The present observations do not allow us to determine the relative contribution of the FAB ions to diffuse ions. Since scattering is limited to a region of several $R_{E}$ from the foreshock 
boundary it is expected that this contribution is small deeper in the foreshock region where connection along the magnetic field is to the more parallel bow shock. If the contribution of scattered FAB ions to diffuse ions is larger near the foreshock boundary one may expect a variation of the relative contribution of alpha particles to diffuse ions with distance from the foreshock boundary.

In summary, the present results show that the scattering of the FAB is leading to the presence of toroidal gyrating ions upstream of the quasi-parallel bow shock. The phase of transformation of a FAB ion population to an intermediate, and later into a toroidal gyrating ion population is correlated with the distance from the FAB. The process takes place in the presence of higher-energy diffuse ions which are shock-accelerated at the quasi-parallel side of the bow shock. Despite the fact that the lower-energy, former FAB ions and the higher-energy, diffuse ions appear together, they have a completely different origin. This might confuse the proper understanding of the physical processes if studies are only based on observations of a single spacecraft or several spacecraft which are relatively close to each other compared with the dimensions of the foreshock region. Therefore it is necessary to analyze also the origin and the evolution of the ion distributions at different energies and their relation to the foreshock geometrical structure.

Acknowledgements. We are grateful to C. W. Smith for the use of the ACE magnetic field data. We should like to thank both referees for their helpful comments and suggestions. The CIS experiment on Cluster at MPE is supported by the German Bundesministerium für Bildung und Forschung and the Zentrum für Luft- und Raumfahrt under contracts 50 OC 0102 and 50OC0001. The project was in part supported by the Hungarian Research Fund, project number NI 61013.

Topical Editor I. A. Daglis thanks G. Zastenker and another referee for their help in evaluating this paper.

\section{References}

Archer, M., Horbury, T. S., Lucek, E. A., Mazelle, C., Balogh, A., and Dandouras, I.: Size and shape of ULF waves in the terrestrial foreshock, J. Geophys. Res., 110, A05208, doi:10.1029/2004JA010791, 2005.

Balogh, A., Carr, C. M., Acuna, M. H., Dunlop, M. W., Beek, T. J., Brown, P., Fornacon, K.-H., Georgescu, E., Glassmeier, K.H., Harris, J.,Musmann, G., Oddy, T., and Schwingenschuh, K.: The Cluster Magnetic Field Investigation: overview of in-flight performance and initial results, Ann. Geophys., 19, 1207-1217, 2001, http://www.ann-geophys.net/19/1207/2001/.

Bonifazi, C. and Moreno, G.: Reflected and diffuse ions backstreaming from the Earth's bow shock: 1. Basic properties, J. Geophys. Res., 86, 4397-4404, 1981.

Bonifazi, C. and Moreno, G.: Reflected and diffuse ions backstreaming from the Earth's bow shock: 2. Origin, J. Geophys. Res., 86, 4405-4414, 1981.
Burgess, D.: Simulations of backstreaming ion beams formed at oblique shocks by direct reflection, Ann. Geophys., 5A, 133$145,1987$.

Eastwood, J. P., Balogh, A., Lucek, E. A., Mazelle, C., and Dandouras, I.: Quasi-monochromatic ULF foreshock waves as observed by the four-spacecraft Cluster mission: 1. Statistical properties, J. Geophys. Res., 110, A11219, doi:10.1029/2004JA010618, 2005.

Edmiston, J. P., Kennel, C. F., and Eichler, D.: Escape of heated ions upstream of quasi-parallel shocks, Geophys. Res. Lett., 9, 531-534, 1982.

Fuselier, S. A., Thomsen, M. F., Gosling, J. T., Bame, S. J., and Russell, C. T.: Gyrating and intermediate ion distributions upstream from the Earth's Bow shock, J. Geophys. Res., 91, 91-99, 1986.

Gosling, J. T., Asbridge, J. R., Bame, S. J., Paschmann, G., and Sckopke, N.: Observations of two distinct populations of bow shock ions in the upstream solar wind, Geophys. Res. Lett., 5, 957-960, 1978.

Gosling, J. T., Thomsen, M. F., Bame, S. J., Feldman, W. C., Paschmann, G., and Sckopke, N.: Evidence for specularly reflected ions upstream from the quasi-parallel bow shock, Geophys. Res. Lett., 9, 1333-1336, 1982.

Greenstadt, E. W., Russell, C. T., and Hoppe, M.: Magnetic field orientation and suprathermal ion streams in the Earth's foreshock, J. Geophys. Res., 85, 3473-3479, 1980.

Greenstadt, E. W., Le, G., and Strangeway, R. J.: ULF waves in the foreshock, Adv. Space Res., 15, 71-74, 1995.

Hoppe, M. M., Russel, C. T., Frank, L. A., Eastman, T. E., and Greenstadt, E. W.: Upstream hydromagnetic waves and their association with backstreaming ions populations: ISEE-1 and ISEE-2 observations, J. Geophys. Res., 86, 4471-4492, 1981.

Hoshino, M. and Terasawa, T.: Numerical study of the upstream waves excitation mechanism: 1 . Nonlinear phase bunching of beam ions, J. Geophys. Res., 90, 57-64, 1985.

Ipavich, F. M., Galvin, A. B., Gloeckler, G., Scholer, M., and Hovestadt, D.: A statistical survey of ions observed upstream of the Earth's bow shock: Energy spectra, composition and spatial variations, J. Geophys. Res., 86, 4337-4342, 1981.

Ipavich, F. M., Gosling, J. T., and Scholer, M.: Correlation between the $\mathrm{He} / \mathrm{H}$ ratios in upstream particle events and in the solar wind, J. Geophys. Res., 89, 1501-1507, 1984.

Ipavich, F. M., Gloeckler, G., Hamilton, D. C., Kistler, L. M., and Gosling, J. T.: Protons and alpha particles in field-aligned beams upstream of the bow shock, Geophys. Res. Lett., 15, 1153-1156, 1988.

Kis, A., Scholer, M., Klecker, B., Möbius, E., Lucek, E. A., Rème, H., Bosqued, J. M., Kistler, L. M., and Kucharek, H.: Multi-spacecraft observations of diffuse ions upstream of Earth's bow shock, Geophys. Res. Lett., 31, L20801, doi:10.1029/2004GL020759, 2004.

Kucharek, H., Möbius, E., Scholer, M., Mouikis, C., Kistler, L. M., Horbury, T., Balogh, A., Rème, H., and Bosqued, J. M.: On the origin of field-aligned beams at the quasi-perpendicular bow shock: Multi-spacecraft observations by Cluster, Ann. Geophys., 22, 2301-2308, 2004, http://www.ann-geophys.net/22/2301/2004/.

Meziane, K., Mazelle, C., Wilber, M., LeQueau, D., Eastwood, J. P., Rm̀e, H., Dandouras, I., Sauvaud, J. C., Bosqued, J. M., Parks, G. K., NcCarthy, M., Klecker, B., Korth, A., Bavassano-Cattaneo 
M.-B., Lundin, R., and Balogh, A.: Bow shock specularly reflected ions in the presence of low-frequency electromagnetic waves: a case study, Ann. Geophys., 22, 2325-2335, 2004, http://www.ann-geophys.net/22/2325/2004/.

Möbius, E., Kucharek, H., Mouikis, C., Georgescu, E., Kistler, L. M., Popecki, M. A., Scholer, M., Bosqued, J. M., Rème, H., Carlson, C. W., Klecker, B., Korth, A., Parks, G. K., Sauvaud, J. C., Balsiger, H., Bavassano-Cattaneo, M.-B., Dandouras, I., DiLellis, A. M., Eliasson, L., Formisano, V., Horbury, T., Lennartsson, W., Lundin, R., McCarthy, M., McFadden, J. P., and Paschmann, G.: Observations of the spatial and temporal structure of field-aligned beam and gyrating ring distributions at the quasi-perpendicular bow shock with Cluster CIS, Ann. Geophys., 19, 1411-1420, 2001, http://www.ann-geophys.net/19/1411/2001/.

Oka, M., Terasawa, T., Saito, Y., and Mukai, T.: Field-aligned beam observations at the quasi-perpendicular bow shock: Generation and shock angle dependence, J. Geophys. Res., 110, A05101, doi:10.1029/2004JA010688, 2005.

Paschmann, G., Sckopke, N., Bame, S. J., Asbridge, J. R., Gosling, J. T., Russell, C. T., and Greenstadt E. W.: Association of lowfrequency waves with suprathermal ions in the upstream solar wind, Geophys. Res. Lett., 6, 209-212, 1979.

Paschmann, G., Sckopke, N., Papamastorakis, I., Asbridge, J. R., Bame, S. J., and Gosling, J. T.: Energetization of solar wind ions by reflection from the earth's bow shock, J. Geophys. Res., 85, 4689-4693, 1980.

Paschmann, G., Sckopke, N., Papamastorakis, I., Asbridge, J. R., Bame, S. J., and Gosling, J. T.: Characteristics of reflected and diffuse ions upstream from the Earth's bow shock, J. Geophys. Res., 86, 4355-4364, 1981.

Paschmann, G., Sckopke, N., Bame, S. J., and Gosling, J. T.: Observations of gyrating ions in the foot of the nearly perpendicular bow shock, Geophys. Res. Lett., 9, 88-91, 1982.

Peredo, M., Slvin, J. A., Mzur, E., and Curtis, S. A.: Threedimensional position and shape of the bow shock and their variation with Alfvénic, sonic, and magnetosonic Mach numbers and interplanetary magnetic field orientation, J. Geophys. Res., 100, 7907-7916, 1995.

Rème, H. , Aoustin, C., Bosqued, J. M., Dandouras, I., Lavraud, B., Sauvaud, J. A., Barthe, A., Bouyssou, J., Camus, Th., CoeurJoly, O., Cros, A., Cuvilo, J., Ducay, F., Garbarowitz, Y., Medale, J. L., Penou, E., Perrier, H., Romefort, D., Rouzaud, J., Vallat, C., Alcaydé, D., Jacquey, C., Mazelle, C., d’Uston, C., Möbius, E., Kistler, L. M., Crocker, K., Granoff, M., Mouikis, C., Popecki, M., Vosbury, M., Klecker, B., Hovestadt, D., Kucharek, H., Kuenneth, E., Paschmann, G., Scholer, M., Sckopke, N., Seidenschwang, E., Carlson, C. W., Curtis, D. W., Ingraham, C., Lin, R. P., McFadden, J. P., Parks, G. K., Phan, T., Formisano, V., Amata, E., Bavassano-Cattaneo, M. B., Baldetti, P., Bruno, R., Chionchio, G., Di Lellis, A., Marcucci, M. F., Pallocchia, G., Korth, A., Daly, P. W., Graeve, B., Rosenbauer, H., Vasyliunas, V., McCarthy, M., Wilber, M., Eliasson, L., Lundin, R., Olsen, S., Shelley, E. G., Fuselier, S., Ghielmetti, A. G., Lennartsson, W., Escoubet, C. P., Balsiger, H., Friedel, R., Cao, J-B., Kovrazhkin, R. A., Papamastorakis, I., Pellat, R., Scudder, J., and Sonnerup, B.: First multispacecraft ion measurements in and near the Earth's magnetosphere with the identical Cluster Ion Spectrometry (CIS) experiment, Ann. Geophys., 19, 1303-1354, 2001, http://www.ann-geophys.net/19/1303/2001/.

Russell, C. T. and Farris, M. H.: Ultra-low frequency waves at the Earth's bow shock, Adv. Space Res., 15, 285-288, 1995.

Scholer, M., Ipavich, F. M., and Gloeckler, G.: Beams of protons and alpha particles greater than approximately $30 \mathrm{keV} / \mathrm{charge}$ from the earth's bow shock, J. Geophys. Res., 86, 4374-4378, 1981.

Schwartz, S. J., Thomsen, M. F., and Gosling, J. T.: Ions upstream of the earth's bow shock: A theoretical comparison of alternative source populations, J. Geophys. Res., 88, 2039-2047, 1983.

Sonnerup, B. U. Ö.: Acceleration of particles reflected at a shock front, J. Geophys. Res., 74, 1301-1304, 1969.

Tanaka, M., Goodrich, C. C., Winske, D., and Papadopoulos, K.: A source of the backstreaming ion beams in the foreshock region, J. Geophys. Res., 88, 3046-3054, 1983.

Thomsen, M. F., Gosling, J. T., Bame, S. J., Feldman, W. C., Paschmann, G., and Sckopke, N.: Field-aligned ion beams upstream of the Earth's bow shock: Evidence for a magnetosheath source, Geophys. Res. Lett., 10, 1207-1210, 1983.

Thomsen, M. F., Gosling, J. T., Bame, S. J., and Russell, C. T.: Gyrating ions and large-amplitude monochromatic MHD waves upstream of the Earth's bow shock, J. Geophys. Res., 90, 267$273,1985$.

Thomsen, M. F.: Upstream suprathermal ions, edited by: Tsurutani, B. T., Stone, R. G., Collisionless Shocks in the Heliosphere: Reviews of Current Research, Geophysics Monography Series, vol. 35, AGU, Washington, D.C., p. 253, 1985. 